\title{
A natural Galois connection between generalized norms and metrics
}

\author{
Árpád Száz \\ Department of Mathematics, \\ University of Debrecen, \\ H-4002 Debrecen, Pf. 400, Hungary \\ email: szaz@science.unideb.hu
}

\begin{abstract}
Having in mind a well-known connection between norms and metrics on vector spaces, for an additively written group $X$, we establish a natural Galois connection between functions of $X$ to $\mathbb{R}$ and $X^{2}$ to $\mathbb{R}$.
\end{abstract}

\section{Introduction}

In this paper, for an additively written group $X$, we shall consider the sets

$$
\mathcal{N}=\mathcal{N}(\mathrm{X})=\mathbb{R}^{\mathrm{X}} \quad \text { and } \quad \mathcal{M}=\mathcal{M}(\mathrm{X})=\mathbb{R}^{\mathrm{X}^{2}} .
$$

to be equipped with the usual pointwise inequality of real-valued functions.

Moreover, having in mind a well-known connection between norms and metrics on vector spaces, for any $p \in \mathcal{N}, \mathrm{d} \in \mathcal{M}$ and $x, y \in X$ we define

$$
p_{d}(x)=d(0, x) \quad \text { and } \quad d_{p}(x, y)=p(-x+y) .
$$

Thus, it can be easily seen that, for any $p \in \mathcal{N}$ and $d \in \mathcal{M}$,
(1) $d_{p} \leq d \Longrightarrow p \leq p_{d}$,
(2) $\mathrm{p} \leq \mathrm{p}_{\mathrm{d}} \Longrightarrow \mathrm{d}_{\mathrm{p}} \leq \mathrm{d}_{\mathrm{p}_{\mathrm{d}}}$.

2010 Mathematics Subject Classification: O6A15, 20A99, 54E25

Key words and phrases: groups, preseminorms, invariant semimetrics, Galois connections 
Moreover, if in particular

$$
\mathrm{d}(\mathrm{x}, \mathrm{y})=|\varphi(\mathrm{x})-\varphi(\mathrm{y})|, \quad \text { with } \quad \varphi(x)=x /(1+|x|),
$$

for all $x, y \in \mathbb{R}$, then $d$ is a metric on $\mathbb{R}$ such that $d_{\mathfrak{p}_{\mathrm{d}}} \leq \mathrm{d}$, despite that $p=p_{d_{p}}$ for all $p \in \mathcal{N}$.

Therefore, by defining

$$
\mathcal{M}^{\wedge}=\mathcal{M}^{\wedge}(\mathrm{X})=\left\{\mathrm{d} \in \mathcal{M}(\mathrm{X}): \quad \mathrm{d}_{\mathrm{p}_{\mathrm{d}}} \leq \mathrm{d}\right\},
$$

we can note that the functions, defined by

$$
f(p)=d_{p} \quad \text { and } \quad g(d)=p_{d}
$$

for all $p \in \mathcal{N}$ and $d \in \mathcal{M}^{\wedge}$, establish an increasing Galois connection [21, 24] between the posets $\mathcal{N}$ and $\mathcal{M}^{\wedge}$ in the sense that, for any $p \in \mathcal{N}$ and $\mathrm{d} \in \mathcal{M}^{\wedge}$, we have

$$
\mathrm{f}(\mathrm{p}) \leq \mathrm{d} \Longleftrightarrow \mathrm{p} \leq \mathrm{g}(\mathrm{d}) .
$$

Some very particular Galois connections have also been investigated in Lambek [12] and our former papers [17, 18, 20, 3, 23, 25, 26]. However, to get a proper overview on Galois connections, the interested reader must consult most of the books $[1,2,9,7,4,5]$.

To feel the importance of our present Galois connection, note that if in particular $p \in \mathcal{N}$ is a preseminorm $[16,28]$ on $X$ in the sense that
(1) $p(0) \leq 0$,
(2) $p(-x) \leq p(x)$,
(3) $p(x+y) \leq p(x)+p(y)$

for all $x, y \in X$, then $d_{p}$ is a left-invariant semimetric on $X$ such that

$$
d(p(x), p(y))=|p(x)-p(y)| \leq d_{p}(x, y)
$$

for all $x, y \in X$.

Conversely, if $d$ is a left-invariant semimetric on $X$, then $p_{d}$ is a preseminorm on $X$ such that $d=d_{p_{d}}$. Therefore, preseminorms and left-invariant semimetrics are equivalent tools in a group. However, in contrast to the opinions of several authors, the former ones, being a function of only one variable, are certainly more convenient tools than the latter ones.

In this respect, it is also worth mentioning that if in particular $d$ is the postman metric $[22]$ on $\mathbb{C}$, i.e.,

$$
d(x, y)=0 \quad \text { if } \quad x=y \quad \text { and } \quad d(x, y)=|x|+|y| \quad \text { if } \quad x \neq y .
$$

for any $x, y \in \mathbb{C}$, then $d$ is a metric on $\mathbb{C}$ such that $d \in \mathcal{M}^{\wedge}(\mathbb{C})$, but $\mathrm{d} \neq \mathrm{d}_{\mathrm{p}_{\mathrm{d}}}$. 


\section{Generalized norms and metrics}

Notation 1 In the sequel, we shall assume that $X$ is an additively written group, and we shall write

$$
\mathcal{N}=\mathcal{N}(\mathrm{X})=\mathbb{R}^{\mathrm{X}} \quad \text { and } \quad \mathcal{M}=\mathcal{M}(\mathrm{X})=\mathbb{R}^{\mathrm{X}^{2}} .
$$

Moreover, on the sets $\mathcal{N}$ and $\mathcal{M}$ we shall consider the usual pointwise inequality of real-valued functions.

Remark 1 Thus, for instance, $\mathcal{N}$ is the space of all functions of $X$ to $\mathbb{R}$. Moreover, since $X^{2}$ is also a group, we can note that $\mathcal{M}(X)=\mathcal{N}\left(X^{2}\right)$.

The members of the families $\mathcal{N}$ and $\mathcal{M}$ may be considered as certain generalized norms and metrics on $X$, respectively. They can be easily connected by the following

Definition 1 For any $d \in \mathcal{M}, p \in \mathcal{N}$ and $x, y \in X$, we define

$$
p_{d}(x)=d(0, x) \quad \text { and } \quad d_{p}(x, y)=p(-x+y) .
$$

Remark 2 Moreover, for any $\mathrm{p} \in \mathcal{N}$ and $\mathrm{d} \in \mathcal{M}$, we also define

$$
f(p)=d_{p} \quad \text { and } \quad g(d)=p_{d} .
$$

Thus, the functions $f$ and $g$ establish a natural connection between $\mathcal{N}$ and $\mathcal{M}$.

By Definition 1, we evidently have the following

Theorem 1 For any $\mathrm{p}, \mathrm{q} \in \mathcal{N}$ and $\mathrm{d}, \rho \in \mathcal{M}$,
(1) $\mathrm{p} \leq \mathrm{q} \Longrightarrow \mathrm{d}_{\mathrm{p}} \leq \mathrm{d}_{\mathrm{q}}$,
(2) $d \leq \rho \Longrightarrow p_{d} \leq p_{\rho}$.

Remark 3 Thus, by Remark 2, for any $p, q \in \mathcal{N}$ and $d, \rho \in \mathcal{M}$
(1) $\mathrm{p} \leq \mathrm{q} \Longrightarrow \mathrm{f}(\mathrm{p}) \leq \mathrm{f}(\mathrm{q})$,
(2) $d \leq \rho \Longrightarrow g(d) \leq g(\rho)$.

Therefore, the functions $f$ and $g$ are increasing.

Moreover, by using Definition 1, we can also easily prove the following

Theorem 2 For any $\mathrm{p} \in \mathcal{N}$, we have
(1) $p=p_{d_{p}}$,
(2) $d_{p}=d_{p_{d p}}$. 
Proof. For any $x \in X$, we have

$$
p_{d_{p}}(x)=d_{p}(0, x)=p(-0+x)=p(x) .
$$

Therefore, $d_{\mathfrak{p}_{\mathfrak{p}}}=p$, and thus (1) is true. Assertion (2) follows from (1).

Remark 4 By Theorem 2 and Remark 2, for any $p \in \mathcal{N}$ we have
(1) $p=g(f(p))$,
(2) $f(p)=f(g(f(p)))$.

Hence, we at once see that $f$ is injective and $g$ maps the range of $f$ onto $\mathcal{N}$. Moreover, $g \circ f$ and $f \circ g$ are the identity functions of $\mathcal{N}$ and $f[\mathcal{N}]$, respectively.

Now, as an immediate consequence of Theorems 1 and 2, we can also state

Theorem 3 For any $\mathrm{p} \in \mathcal{N}$ and $\mathrm{d} \in \mathcal{M}$,
(1) $\mathrm{d}_{\mathrm{p}} \leq \mathrm{d} \Longrightarrow \mathrm{p} \leq \mathrm{p}_{\mathrm{d}}$
(2) $p \leq p_{d} \Longrightarrow d_{p} \leq d_{p_{d}}$.

Proof. To prove (1), note that if $d_{p} \leq d$ holds, then by Theorem 1 we also have $p_{d_{p}} \leq p_{d}$. Moreover, by Theorem 2, we have $p_{d_{p}}=p$. Therefore, $p \leq p_{d}$ also holds.

Remark 5 By Theorem 3 and Remark 2, for any $p \in \mathcal{N}$ and $\mathrm{d} \in \mathcal{M}$
(1) $f(p) \leq d \Longrightarrow p \leq g(d)$,
(2) $p \leq g(d) \Longrightarrow f(p) \leq f(g(d))$.

\section{Three important subfamilies of $\mathcal{M}$}

Because of Theorem 3, we may naturally introduce the following

Definition 2 Define

$$
\begin{array}{ll}
\mathcal{M}^{*}=\mathcal{M}^{*}(X)=\{d \in \mathcal{M}(X): & \left.d=d_{p_{d}}\right\}, \\
\mathcal{M}^{\wedge}=\mathcal{M}^{\wedge}(X)=\{d \in \mathcal{M}(X): & \left.d_{\mathfrak{p}_{d}} \leq d\right\}, \\
\mathcal{M}^{\vee}=\mathcal{M}^{\vee}(X)=\{d \in \mathcal{M}(X): & \left.d \leq d_{p_{d}}\right\} .
\end{array}
$$


Remark 6 Thus, by Remark 2, we have

$$
\begin{array}{ll}
\mathcal{M}^{*}=\mathcal{M}^{*}(\mathrm{X})=\{\mathrm{d} \in \mathcal{M}: & \mathrm{d}=\mathrm{f}(\mathrm{g}(\mathrm{d}))\}, \\
\mathcal{M}^{\wedge}=\mathcal{M}^{\wedge}(\mathrm{X})=\{\mathrm{d} \in \mathcal{M}: & \mathrm{f}(\mathrm{g}(\mathrm{d})) \leq \mathrm{d}\}, \\
\mathcal{M}^{\vee}=\mathcal{M}^{\vee}(\mathrm{X})=\{\mathrm{d} \in \mathcal{M}: & \mathrm{d} \leq \mathrm{f}(\mathrm{g}(\mathrm{d}))\} .
\end{array}
$$

The importance of the family $\mathcal{M}^{\wedge}$ is already quite obvious from the following

Theorem 4 For any $\mathrm{d} \in \mathcal{M}$, the following assertions are equivalent:
(1) $\mathrm{d} \in \mathcal{M}^{\wedge}$,
(2) $\mathrm{p} \leq \mathrm{p}_{\mathrm{d}} \Longrightarrow \mathrm{d}_{\mathrm{p}} \leq \mathrm{d}$ for all $\mathrm{p} \in \mathcal{N}$.

Proof. If $p \in \mathcal{N}$ and $p \leq p_{d}$, then by Theorem 1 we have $d_{p} \leq d_{p_{d}}$. Moreover, if in particular (1) holds, then by Definition 2 we have $d_{p_{d}} \leq d$. Therefore, if (1) holds, then $d_{\mathfrak{p}} \leq \mathrm{d}$, and thus (2) also holds.

Conversely, if (2) holds, the from the trivial inequality $p_{d} \leq p_{d}$ we can already infer that $d_{p_{d}} \leq d$. Thus, by Definition 2, (1) also holds.

Remark 7 By Theorem 4 and Remark 2, for any $\mathrm{d} \in \mathcal{M}$ the following assertions are equivalent :

(1) $\mathrm{d} \in \mathcal{M}^{\wedge}$, (2) $p \leq g(d) \Longrightarrow f(p) \leq d$ for all $p \in \mathcal{N}$.

Now, as an immediate consequence of Theorems 3 and 4, we can also state Theorem 5 For any $\mathrm{p} \in \mathcal{N}$ and $\mathrm{d} \in \mathcal{M}^{\wedge}$, we have

$$
\mathrm{d}_{\mathrm{p}} \leq \mathrm{d} \quad \Longleftrightarrow \mathrm{p} \leq \mathrm{p}_{\mathrm{d}} .
$$

Remark 8 Thus, by Remark 2 , for any $p \in \mathcal{N}$ and $d \in \mathcal{M}^{\wedge}$ we have

$$
f(p) \leq d \quad \Longleftrightarrow p \leq g(d) .
$$

This shows that the function $f$ and the restriction of $g$ to $\mathcal{M}^{\wedge}$ form an increasing Galois connection $[19,21,24]$ between the posets $\mathcal{N}$ and $\mathcal{M}^{\wedge}$.

Thus, several consequences of Definition 1 can be immediately derived from the theory of Galois connections $[1,2,9,7,4]$.

However, because of the simplicity of Definition 1, it seems now more convenient to apply some direct proofs. 
For instance, by using Definition 2 and Theorem 2, we can easily prove

Theorem 6 For any $\mathrm{d} \in \mathcal{M}$, the following assertions are equivalent:
(1) $d \in \mathcal{M}^{*}$,
(2) $\mathrm{d}=\mathrm{d}_{\mathrm{p}}$ for some $\mathrm{p} \in \mathcal{N}$.

Proof. If (1) holds, then by Definition 2 we have $d=d_{\mathfrak{p}_{d}}$. Therefore, (2) also holds with $p=p_{d}$.

Moreover, by Theorem 2, we have $d_{\mathfrak{p}}=d_{\mathfrak{p}_{\mathfrak{d}}}$, and thus by Definition 2 $d_{\mathfrak{p}} \in \mathcal{M}^{*}$ for all $p \in \mathcal{N}$. Therefore, if (2) holds, then (1) also holds.

Remark 9 From Theorem 6, by Remark 2, we can see that $\mathcal{M}^{*}=\mathrm{f}[\mathcal{N}]$.

\section{Some further characterizations of $\mathcal{M}^{\wedge}$ and $\mathcal{M}^{*}$}

In addition to Theorem 4, we can also prove the following

Theorem 7 For any $\mathrm{d} \in \mathcal{M}$, the following assertions are equivalent:

(1) $\mathrm{d} \in \mathcal{M}^{\wedge}$,

(2) $d(0, y) \leq d(x, x+y)$ for all $x, y \in X$,

(3) $d(0,-x+y) \leq d(x, y)$ for all $x, y \in X$.

Proof. By Definition 2, (1) means only that $d_{p_{d}} \leq d$. That is,

$$
d_{p_{d}}(x, y) \leq d(x, y)
$$

for all $x y \in X$. Hence, by using that

$$
d_{p_{d}}(x, y)=p_{d}(-x+y)=d(0,-x+y)
$$

for all $x, y \in X$, we can see that (1) and (3) are equivalent.

Moreover, if (3) holds, then by writing $x+y$ in place of $y$, we can see that (2) also holds. While, if (2) holds, then by writing $-x+y$ in place of $y$ we can see that (3) also holds.

Analogously to Theorem 7, we can also prove the following

Theorem 8 For any $\mathrm{d} \in \mathcal{M}$, the following assertions are equivalent:

(1) $\mathrm{d} \in \mathcal{M}^{\vee}$,

(2) $\mathrm{d}(x, x+y) \leq \mathrm{d}(0, y)$ for all $x, y \in X$,

(3) $d(x, y) \leq d(0,-x+y)$ for all $x, y \in X$. 
Now, by using that $\mathcal{M}^{*}=\mathcal{M}^{\wedge} \cap \mathcal{M}^{\vee}$, we can also prove the following

Theorem 9 For any $\mathrm{d} \in \mathcal{M}$, the following assertions are equivalent:

(1) $\mathrm{d} \in \mathcal{M}^{*}$,

(2) $d(0, y)=d(x, x+y)$ for all $x, y \in X$,

(3) $d(x, y)=d(0,-x+y)$ for all $x, y \in X$,

(4) $\mathrm{d}(x, y)=\mathrm{d}(z+x, z+y)$ for all $x, y, z \in X$,

(5) $\mathrm{d}(x, y) \leq \mathrm{d}(z+x, z+y)$ for all $x, y, z \in X$,

(6) $\mathrm{d}(z+x, z+y) \leq \mathrm{d}(x, y)$ for all $x, y, z \in X$.

Proof. By Theorems 7 and 8, it is clear that (1), (2) and (3) are equivalent. Moreover, if (4) holds, then by writing $-x$ in place of $z$ we can see that (3) also holds.

While, if (3) holds, then we have

$$
\begin{aligned}
\mathrm{d}(z+x, z+y)=d & (0,-(z+x)+z+y) \\
& =d(0,-x-z+z+y)=d(0,-x+y)=d(x, y)
\end{aligned}
$$

for all $x, y, z \in X$. Therefore, (4) also holds.

Now, since (4) trivially implies (5) and (6), it remains to show only that that (5) and (6) also imply (4). For this, note that if for instance (6) holds, then by writing $-z+x$ in place of $x$ and $-z+y$ in place of $y$, we obtain

$$
d(x, y) \leq d(-z+x,-z+y)
$$

for all $x, y, z \in X$. Hence, by writing $-z$ in place of $z$, we can see that (5) also holds. Therefore, we actually have (4).

Remark 10 The above theorem shows that $\mathcal{M}^{*}$ is just the family of all left-invariant members of $\mathcal{M}$.

Moreover, by using Theorem 9, we can also prove the following

Theorem 10 For a symmetric member $\mathrm{d}$ of $\mathcal{M}$, the following assertions are also equivalent:

(1) $\mathrm{d} \in \mathcal{M}^{*}$

(2) $\mathrm{d}(x, 0)=\mathrm{d}(z+x, z)$ for all $x, z \in X$. 
Proof. If (1) holds, then from (4) in Theorem 9, by taking $y=0$, we can at once see that (2) also holds even if $\mathrm{d}$ is not assumed to be symmetric.

While, if (2) holds, then by using the symmetry of $d$, we can see that

$$
d(0, y)=d(y, 0)=d(x+y, x)=d(x, x+y)
$$

for all $x, y \in X$. Therefore, by Theorem 9, assertion (1) also holds.

\section{$5 \quad$ Two illustrating particular metrics}

Theorem 11 Suppose that $\mathrm{X}$ is a normed space such that

$$
\|u+v\|<\|u\|+\|v\|
$$

for some $\mathrm{u}, \boldsymbol{v} \in \mathrm{X}$ with $\mathrm{u}+\boldsymbol{v} \neq 0$. And, for any $\mathrm{x}, \mathrm{y} \in \mathrm{X}$, define

$$
d(x, y)=0 \quad \text { if } \quad x=y \quad \text { and } \quad d(x, y)=\|x\|+\|y\| \quad \text { if } \quad x \neq y .
$$

Then, $\mathrm{d}$ is a metric on $\mathrm{X}$ such that

$$
\mathrm{d} \in \mathcal{M}^{\wedge}(\mathrm{X}) \backslash \mathcal{M}^{\vee}(\mathrm{X}) .
$$

Proof. To prove the latter statement, note that, for any $x, y \in X$ with $y \neq 0$, we have

$$
\mathrm{d}(0, y)=\|y\|=\|-x+x+y\| \leq\|x\|+\|x+y\|=d(x, x+y) .
$$

Hence, since $d(0,0) \leq d(x, x)$ trivially holds, by Theorem 7 we can see that $\mathrm{d} \in \mathcal{M}^{\wedge}(\mathrm{X})$.

Moreover, note that for $x=-u$ and $y=u+v$ we have $\mathrm{d}(0, y)=\|\mathrm{y}\|=\|\mathrm{u}+v\|<\|u\|+\|v\|=\|x\|+\|x+y\|=\mathrm{d}(x, x+y)$. Therefore, by Theorem $8, \mathrm{~d} \notin \mathcal{M}^{\vee}(\mathrm{X})$ also holds.

Remark 11 To be more concrete, note that if for instance $X=\mathbb{R}^{2}$, and moreover $u=(1,0)$ and $v=(0,1)$, then

$$
u+v=(1,1) \neq(0,0) \quad \text { and } \quad\|u+v\|=\sqrt{2}<2=\|u\|+\|v\| .
$$


Theorem 12 Suppose that $\varphi$ is an injective function of a group $\mathrm{X}$ to a normed space $\mathrm{Y}$. And, for any $\mathrm{x}, \mathrm{y} \in \mathrm{X}$, define

$$
\mathrm{d}(\mathrm{x}, \mathrm{y})=\|\varphi(\mathrm{x})-\varphi(\mathrm{y})\| \text {. }
$$

Then, $\mathrm{d}$ is a metric on $\mathrm{X}$ such that

(1) $\mathrm{d} \in \mathcal{M}^{\wedge}(\mathrm{X})$ if and only if $\|\varphi(\mathrm{y})-\varphi(0)\| \leq\|\varphi(x+y)-\varphi(x)\|$ for all $x, y \in X$,

(2) $\mathrm{d} \in \mathcal{M}^{\vee}(\mathrm{X})$ if and only if $\|\varphi(x+y)-\varphi(x)\| \leq\|\varphi(y)-\varphi(0)\|$ for all $x, y \in X$.

Proof. To prove (1), note that by Theorem 7 and the definition of $d$ we have $\mathrm{d} \in \mathcal{M}^{\wedge}(\mathrm{X})$ if and only if

$\mathrm{d}(0, y) \leq \mathrm{d}(\mathrm{x}, \mathrm{x}+\mathrm{y}), \quad$ i. e., $\quad\|\varphi(0)-\varphi(x)\| \leq\|\varphi(x)-\varphi(x+y)\|$ for all $x, y \in X$. Therefore, (1) is true.

Now, as an immediate consequence of this theorem, we can also state

Corollary 1 Under the assumptions of Theorem 12, we have $\mathrm{d} \in \mathcal{M}^{*}(\mathrm{X})$ if and only if $\|\varphi(\mathrm{x}+\mathrm{y})-\varphi(\mathrm{x})\|=\|\varphi(\mathrm{y})-\varphi(0)\|$ for all $\mathrm{x}, \mathrm{y} \in \mathrm{X}$.

Remark 12 Note that in the above results, because of

$$
\mathrm{d}(\mathrm{x}, \mathrm{y})=\|\varphi(x)-\varphi(\mathrm{y})\|=\|\varphi(x)-\varphi(0)-(\varphi(y)-\varphi(0))\|,
$$

we may assume, without a genuine loss of generality, that $\varphi(0)=0$.

Moreover, by using the notation

$$
\Delta_{y} \varphi(x)=\varphi(x+y)-\varphi(x)
$$

for all $x, y \in X$, the definition of $d$ and the condition of Corollary 1 can be reformulated in the forms that

$\mathrm{d}(x, y)=\left\|\Delta_{x} \varphi(0)-\Delta_{y} \varphi(0)\right\| \quad$ and $\quad\left\|\Delta_{y} \varphi(0)\right\|=\min _{x \in X}\left\|\Delta_{y} \varphi(x)\right\|$ for all $x, y \in X$.

From Corollary 1, it is clear that in particular we also have 
Corollary 2 If in addition to the assumptions of Theorem 12, the function $\varphi$ is additive, then $\mathrm{d} \in \mathcal{M}^{*}(\mathrm{X})$.

Remark 13 In this respect, it is noteworthy that if $\varphi$ is a function of a group $\mathrm{X}$ to a normed space $\mathrm{Y}$ such that

$$
\|\varphi(x+y)-\varphi(x)\| \leq\|\varphi(y)\|
$$

for all $x, y \in X$, then by writing $-u$ in place of $x$ and $u+v$ in place of $y$ we obtain

$$
\|\varphi(v)-\varphi(-\mathfrak{u})\| \leq\|\varphi(u+v)\|
$$

for all $u, v \in X$.

Therefore, if in particular $\varphi$ is odd, then we have

$$
\|\varphi(u)+\varphi(v)\| \leq\|\varphi(u+v)\|
$$

for all $u, v \in X$. (Note that the latter property already implies that $\varphi(0)=0$ and $\varphi$ is odd.)

Moreover, if in particular $\mathrm{Y}$ is an inner product space, then by a basic theorem of Maksa and Volkmann [14], we can state that $\varphi$ is additive. (For some closely related results, see $[6,11,15,29,30,8,27,28]$.)

Concerning Theorem 12, it is also worth mentioning that Makai [13] proved that there exists a nowhere continuous additive function $\varphi$ of $\mathbb{R}$ to itself such that $\varphi=\varphi^{-1}$. (For a more general result, see Kuzcma [10, p. 293] .)

However, it is now more important to note that, by using Theorem 12, we can also prove the following

Theorem 13 If $\varphi$ is an injective function of $\mathbb{R}$ to a normed space $\mathrm{Y}$ such that

$$
\lim _{x \rightarrow-\infty} \varphi(x)=\alpha \quad \text { and } \quad \lim _{x \rightarrow+\infty} \varphi(x)=\beta
$$

with $\alpha, \beta \in \mathrm{Y}$ such that $\|\alpha-\varphi(0)\|<\|\alpha-\beta\|$, and

$$
d(x, y)=\|\varphi(x)-\varphi(y)\|
$$

for all $x, y \in \mathbb{R}$, then $\mathrm{d}$ is a metric on $\mathbb{R}$ such that

$$
\mathrm{d} \notin \mathcal{M}^{\wedge}(\mathbb{R}) \cup \mathcal{M}^{\vee}(\mathbb{R}) .
$$


Proof. To prove the latter statement, note that if $d \in \mathcal{M}^{\wedge}(\mathbb{R})$, then by Theorem 12 we have

$$
\|\varphi(y)-\varphi(0)\| \leq\|\varphi(x+y)-\varphi(x)\|
$$

for all $x, y \in \mathbb{R}$. Hence, by letting $x$ tend to $+\infty$, we can infer that

$$
\|\varphi(y)-\varphi(0)\| \leq 0,
$$

and thus $\varphi(y)=\varphi(0)$ for all $y \in \mathbb{R}$. This contradiction proves that $\mathrm{d} \notin$ $\mathcal{M}^{\wedge}(\mathbb{R})$.

While, if $\mathrm{d} \in \mathcal{M}^{\vee}(\mathbb{R})$, then by Theorem 12 we have

$$
\|\varphi(x+y)-\varphi(x)\| \leq\|\varphi(y)-\varphi(0)\|
$$

for all $x, y \in \mathbb{R}$. Hence, by letting $y$ tend to $-\infty$, we can infer that

$$
\|\alpha-\varphi(x)\| \leq\|\alpha-\varphi(0)\|,
$$

for all $x \in \mathbb{R}$. Hence, by letting $x$ tend to $+\infty$, we can infer that

$$
\|\alpha-\beta\| \leq\|\alpha-\varphi(0)\| \text {. }
$$

This contradiction proves that $\mathrm{d} \notin \mathcal{M}^{\vee}(\mathbb{R})$.

Remark 14 To be more concrete, note that if for instance

$$
\varphi(x)=x /(1+|x|)
$$

for all $x \in \mathbb{R}$, then $\varphi$ is a strictly increasing function of $\mathbb{R}$ to itself such that $\varphi(0)=0$,

$$
\alpha=\lim _{x \rightarrow-\infty} \varphi(x)=-1 \quad \text { and } \quad \beta=\lim _{x \rightarrow+\infty} \varphi(x)=1 .
$$

Therefore, $|\alpha-\varphi(0)|=1<2=|\alpha-\beta|$.

\section{Acknowledgement}

The work of the author has been supported by the Hungarian Scientific Research Fund (OTKA) Grant K-111651.

Moreover, the author is indebted to Zsolt Páles for an observation leading to some shortenings of the proofs in Section 2. 


\section{References}

[1] G. Birkhoff, Lattice Theory, Amer. Math. Soc. Colloq. Publ., 25, Providence, RI, 1967.

[2] T.S. Blyth, M.F. Janowitz, Residuation Theory, Pergamon Press, Oxford, 1972.

[3] S. Buglyó, Á. Száz, A more important Galois connection between distance functions and inequality relations, Sci. Ser. A Math. Sci. (N.S.), 18 (2009), 17-38.

[4] B. A. Davey, H. A. Priestley, Introduction to Lattices and Order, Cambridge University Press, Cambridge, 2002.

[5] K. Denecke, M. Erné, S. L. Wismath (Eds.) Galois Connections and Applications, Kluwer Academic Publisher, Dordrecht, 2004.

[6] P. Fischer, Gy. Muszély, On some new generalizations of the functional equation of Cauchy, Canadian Math. Bull., 10 (1967), 197-205.

[7] B. Ganter, R. Wille, Formal Concept Analysis, Springer-Verlag, Berlin, 1999.

[8] R. Ger, Fischer-Muszély additivity on Abelian groups, Comment Math., Tomus Specialis in Honorem Juliani Musielak (2004), 83-96.

[9] G. Gierz, K. H. Hofmann, K. Keimel, J. D. Lawson, M. Mislove, D. S. Scott, A Compendium of Continuous Lattices, Springer-Verlag, Berlin, 1980.

[10] M. Kuczma, An Introduction to the Theory of Functional Equations and Inequalities, Państwowe Wydawnictwo Naukowe, Warszawa, 1985.

[11] S. Kurepa, On P. Volkmann's paper, Glasnik Mat., 22 (1987), 371-374.

[12] J. Lambek, Some Galois connections in elementary number theory, $J$. Number Theory, 47 (1994), 371-377.

[13] I. Makai, Über invertierbare Lösungen der additive CauchyFunctionalgleichung, Publ. Math. Debrecen, 16 (1969), 239-243. 
[14] Gy. Maksa, P. Volkmann, Characterizations of group homomorphisms having values in an inner product space, Publ. Math. Debrecen, 56 (2000), 197-200.

[15] P. Schöpf, Solutions of $\|f(\xi+\eta)\|=\|f(\xi)+f(\eta)\|$, Math. Pannon. 8 (1997), 117-127.

[16] Á. Száz, Preseminormed spaces, Publ. Math. Debrecen, 30 (1983), $217-224$.

[17] Á. Száz, A Galois connection between distance functions and inequality relations, Math. Bohem., 127 (2002), 437-448.

[18] Á. Száz, Galois-type connections on power sets and their applications to relators, Tech. Rep., Inst. Math., Univ. Debrecen, 2005/2, 38 pp.

[19] Á. Száz, Supremum properties of Galois-type connections, Comment. Math. Univ. Carolin., 47 (2006), 569-583.

[20] Á. Száz, An instructive treatment of convergence, closure and orthogonality in semi-inner product spaces, Tech. Rep., Inst. Math., Univ. Debrecen, 2006/2, 29 pp.

[21] Á. Száz, Galois type connections and closure operations on preordered sets, Acta Math. Univ. Comen., 78 (2009), 1-21.

[22] Á. Száz, A common generalization of the postman, radial, and river metrics, Rostock. Math. Kolloq., 67 (2012), 89-125.

[23] Á. Száz, A particular Galois connection between relations and set functions, Acta Univ. Sapientiae, Math., 6 (2014), 73-91.

[24] Á. Száz, Galois and Pataki connections on generalized ordered sets, Tech. Rep., Inst. Math., Univ. Debrecen, 2014/3, 27 pp.

[25] Á. Száz, Remarks and Problems at the Conference on Inequalities and Applications, Hajdúszoboszló, Hungary, 2014, Tech. Rep., Inst. Math., Univ. Debrecen, 2014/5, 12 pp.

[26] Á. Száz, The closure-interior Galois connection and its applications to relational inclusions and equations, Tech. Rep., Inst. Math., Univ. Debrecen, 2015/2, 40 pp. 
[27] Á. Száz, Generalization of a theorem of Maksa and Volkmann on additive functions, Tech. Rep., Inst. Math., Univ. Debrecen, 1016/5, 6 pp. (An improved and enlarged version is available from the author.)

[28] Á. Száz, Remarks and problems at the Conference on Inequalities and Applications, Hajdúszoboszló, Hungary, 2016, Tech. Rep., Inst. Math., Univ. Debrecen, 2016/9, 34 pp.

[29] Jacek Tabor, Józef Tabor, 19. Remark (Solution of the 7. Problem posed by K. Nikodem.), Aequationes Math., 61 (2001), 307-309.

[30] Jacek Tabor, Stability of the Fisher-Muszély functional equation, Publ. Math. Debrecen, 62 (2003), 205-211.

Received: February 2, 2017 